\title{
The Novel DPP-BDT Nanoparticles as Efficient Photoacoustic Imaging and Positron Emission Tomography Agents in Living Mice
}

This article was published in the following Dove Press journal: International Journal of Nanomedicine

\author{
Tingting $\mathrm{Li}^{1,2, *}$ \\ Xiaoming $\mathrm{Hu}\left(\mathbb{D D}^{3, *}\right.$ \\ Quli Fan ${ }^{4, *}$ \\ Zejing Chen ${ }^{3}$ \\ Ziliang Zheng' \\ Ruiping Zhang ${ }^{2}$ \\ 'Department of Pharmacy, School of \\ Pharmaceutical Science, Shanxi Medical \\ University, Taiyuan, Shanxi, People's \\ Republic of China; ${ }^{2}$ Radiology \\ Department, The Affiliated Bethune \\ Hospital of Shanxi Medical University, \\ Taiyuan, Shanxi, People's Republic of \\ China; ${ }^{3}$ Institute of Advanced Materials, \\ East China Jiaotong University, Nanchang, \\ Jiangxi, People's Republic of China; ${ }^{4}$ Key \\ Laboratory for Organic Electronics \& \\ Information Displays and Institute of \\ Advanced Materials, Nanjing University of \\ Posts \& Telecommunications, Nanjing, \\ Jiangsu, People's Republic of China
}

*These authors contributed equally to this work
Correspondence: Ruiping Zhang Radiology Department, The Affiliated Bethune Hospital of Shanxi Medical University, Taiyuan, Shanxi, People's Republic of China

Email zrp_7I42@sxmu.edu.cn
Background: Molecular imaging is of great benefit to early disease diagnosis and timely treatment. One of the most striking innovations is the development of multimodal molecular imaging technology, which integrates two or more imaging modalities, largely in view of making the best of the advantages of each modality while overcoming their respective shortcomings. Hence, engineering a versatile and easily prepared nanomaterial with integrating multimodal molecular imaging function holds great promise, but is still a great challenge. Materials and Methods: We firstly designed and synthesized a BDT-DPP conjugated polymer and then noncovalent self-assembly with phospholipid-polyethylene glycol endowed BDT-DPP with water solubility and biocompatibility. Followed by $[\mathrm{Cu}]$ labeling, the acquired multifunctional nanoparticles (NPs) were studied in detail for the photophysical property. The cytotoxicity and biocompatibility of DPP-BDT NPs were examined through MTT assay and H\&E stained analysis. In addition, we investigated the accumulation of the NPs in HepG2 tumor models by positron emission tomography (PET) and photoacoustic (PA) dual-mode imaging.

Results and Discussion: The DPP-BDT NPs exhibited excellent optical stability, strong near-infrared (NIR) light absorption as well as fine biocompatibility. After tail vein injection into the living mice, the PA signals in the neoplastic tissues were gradually increased and reached to the maximum at the 4-h post-injection, which was consistent with the PET analysis. Such strong PA and PET signals were attributed to the efficient NPs accumulation resulting from the enhanced permeability and retention (EPR) effect.

Conclusion: The biocompatible DPP-BDT NPs demonstrated to be strong NIR absorption property and PAI sensitivity. Besides, these novel DPP-BDT NPs can act not only as a PA imaging contrast agent but also as an imaging agent for PET.

Keywords: conjugated polymer, nanoparticles, photoacoustic imaging, positron emission tomography imaging

\section{Introduction}

Precise diagnosis of cancer is the key to modern clinical cancer treatment. ${ }^{1-3}$ The location and size of the tumor and its spreading situation are mainly known through anatomical imaging, such as positron emission tomography (PET), magnetic resonance imaging (MRI), computed tomography (CT), optical imaging (OI) and photoacoustic imaging (PAI) technologies, which help us further acquire molecular and physiological information of the tumors. ${ }^{4-6}$ PET possesses great superiority for diagnosing, identifying and visualizing the stages of numerous kinds of tumours 
and it is also the imaging technology to display the in vivo biodistribution of the contrast agents and the physiopathological information owing to its unique advantages for whole-body imaging. ${ }^{7}$ However, in consideration of the stochastic annihilation events and the tissue scattering of the gamma rays, PET is not convenient for imaging tissues at a high spatial resolution. ${ }^{8}$ Usually, complementation of dual or more kind of imaging modalities provides more accurate and precise information of cancer. ${ }^{9}$ Among various biomedical imaging techniques, PAI is an emerging hybrid and potent imaging technique, which is noninvasive with fine spatial resolution and strong optical absorption contrast of biological structures than other commonly used methods. ${ }^{10,11}$ PAI overcomes the depth limits of conventional fluorescence and bioluminescence imaging techniques, providing effective and molecular information of the tumorous tissue with deep penetration up to $\sim 7 \mathrm{~mm} .^{12}$ Thus far, PAI has been widely adopted in disease tissue detecting applications, such as inflammatory arthritis, evaluations of hepatic function, tumor diagnosis and imaging-guided cancer treatment. ${ }^{13-18}$

The emergence of nanotechnology has opened a new vision for biomedical research. Currently, numerous nearinfrared (NIR) absorption nanomaterials have been served as PAI contrast agents. Some inorganic nanomaterials such as rare-earth-doped materials, $\mathrm{Ag}_{2} \mathrm{~S}$ quantum dots, carbon nanotubes and gold nanoparticles (NPs) have shown fine superiority and performance for in vivo PAI. ${ }^{3,19-22}$ However, these inorganic nanomaterials always suffer from the long-term toxicity concerns, thus largely restricting their further applications. In addition, some conventional small molecular organic dyes like methylene blue (MB) and indocyanine green (ICG), with effective NIR-absorptive properties possess attractive capabilities for PAI. ${ }^{27,28}$ Despite the superiority for in vivo PAI, the inherent optical instability, expensive cost and difficult modification of these small molecular dyes obviously hinder their further developments. ${ }^{29}$ By contrast, conjugated polymer NPs gradually become the ideal candidate for PAI, mainly because of their high $\pi-\pi$ delocalized backbone, thus resulting in the strong NIR-absorption and excellent optical stability. ${ }^{30}$ For example, some conjugated polymers such as Ppy and PEDOT: PSS have been greatly developed in the field of NIR imaging and phototherapy on account of the numerous superiorities containing splendid photothermal conversion efficiency, high molar extinction coefficient, fine physiological environment stability and ease of preparation. ${ }^{31}$ Compared with the FDA-approved ICG, they have higher photostability, which could be hopeful to become a substitute of ICG and used in PAI for imaging-guided therapy and clinical translation. $^{32}$

In this work, we designed and prepared an effective versatile nanoplatform based on conjugated polymer to realize in vivo PAI and PET dual-mode tumor imaging. The conjugated polymer DPP-BDT was successfully designed and conducted with the alkyl chain modified diketopyrrolopyrrole (DPP) and benzodithiophene (BDT) units through Stille coupling polymerization. The amphiphilic molecules DSPE-mPEG5000 was employed to encapsulate the asprepared polymer via self-assembly process. To endow the NPs with PET imaging property, the radionuclide ${ }^{6 \mathrm{Cu}}$ was further labelled to the NPs, affording the final DPP-BDT NPs. The physical properties of the DPP-BDT NPs were studied in detail. In addition, we systematically investigated the DPP-BDT NPs in HepG2 tumors via both PET and PA imaging. After systemic injection of the tumorous mice using DPP-BDT NPs, the neoplastic regions were greatly lighted up, accompanied by the strong PA signals and PET imaging contrast. All in all, the DPP-BDT NPs possessed intrinsic advantages, such as higher biocompatibility, higher photosensitivity and easier preparation. Given its prominent property, DPP-BDT NPs exhibited great promise for further biological applications.

\section{Materials and Methods Materials}

2.5-dioctyl-3,6-bis(5-(trimethylstannyl)thiophen-2-yl)-2,5Dihydropyrrolo[3,4-c]pyrrole-1,4-dione (DPP, 98\%) and 2.6-dibromo-4,8-bis(4,5-dioctylthiophen-2-yl) benzo[1,2b:4,5-b']dithiophene (BDT, 98\%) were bought from Beijing HWRK Chemical Co., Ltd (Beijing, China). N, N-Dimethylformamide (DMF) and dichloromethane (DCM) were dried and distilled under a nitrogen atmosphere before use. DSPE-PEG5000, DSPE-mPEG 5000- $\mathrm{NH}_{2}$, dimethylthiazolyl-diphenyltetrazolium (MTT), agarose, and tetraazacyclododecane-1,4,7,10-tetraacetic acid (DOTA) were obtained from Sigma Aldrich (Shanghai, China). Unless otherwise stated, all starting materials and organic solvents were purchased from commercial suppliers and used without further purification. HepG2 cells and NIH-3T3 cells were purchased from the Shanghai Laboratory Animal Center, Chinese Academy of Sciences (SLACCAS). Dulbecco's modified Eagle's medium (DMEM) was obtained from Gene Tech Co. (Shanghai, China) ${ }^{6 \mathrm{Cu}}$ was produced with an onsite cyclotron (GE PETrace) in Stanford University, and used for the 
radiosynthesis with the chemical form of ${ }^{6 \mathrm{CuCl} 2}$ (radiochemical purity was 95\%). Deionized (DI) water was used for all experimental procedures.

\section{Synthesis of Conjugated Polymer DPP-BDT}

The DPP (19.87 mg, $0.025 \mathrm{mmol})$, compound BDT (24.02 $\mathrm{mg}, 0.025 \mathrm{mmol})$ and $\mathrm{Pd}\left(\mathrm{PPh}_{3}\right)_{4}(6 \mathrm{mg})$ were dissolved in $4 \mathrm{~mL}$ of anhydrous toluene and transferred into a Schlenk tube $(20 \mathrm{~mL})$ under nitrogen atmosphere. The reaction tube was placed at $110^{\circ} \mathrm{C}$ and stirred for 24 h. After the Stille-coupling reaction, the solvent of the system was removed via decompression. Then, the obtained black crude product was repeatedly extracted using the mixing liquid of deionized water and chloroform. Afterword, the collected concentrated organic phase was further precipitated in cold methanol for three times. Finally, the generated black solid was dried in a vacuum drying chamber to afford product conjugated polymer DPP-BDT.

\section{Preparation of DPP-BDT NPs}

The DPP-BDT NPs were prepared through a nanoprecipitation approach. ${ }^{33}$ Shortly, the conjugated polymer DPP-BDT (2.0 mg), DSPE-mPEG5000 (18 mg) and DSPE-mPEG5000 -NH-NOTA ( $2 \mathrm{mg}$ ) were dispersed in $2 \mathrm{~mL}$ of THF. Then, the mixing liquid was quickly dropped into a mixture of THF $(2 \mathrm{~mL})$ and deionized water $(18 \mathrm{~mL})$ under uninterrupted sonication for $2 \mathrm{~min}$. The remanent organic solvent was swept away by nitrogen blowing upon the system surface under stirring at $45^{\circ} \mathrm{C}$. Finally, the concentrated solution was re-dispersed in phosphate buffer solution (PBS) to afford DPP-BDT NPs. The NPs were stored in a refrigerator for further in vitro characterizations and in vivo experiments.

\section{Preparation of Radioactive ${ }^{6 \mathrm{Cu}}$ Labeled DPP-BDT NPs}

To prepare radiometal ${ }^{6 \mathrm{Cu}}$-labeled DPP-BDT NPs, DOTA was conjugated on the DPP-BDT NPs surface according to the reference procedure. Typically, DOTA was activated by EDC and NHS at $\mathrm{pH} 5.5$ for 30 min with a molar ratio of DOTA/EDC/NHS $=10: 5: 4$. The activated DOTA was then was added into $200 \mu \mathrm{L}$ amine-based DPP-BDT NPs and incubated at a $\mathrm{pH}$ of 8.5. The mixture was then incubated at $40^{\circ} \mathrm{C}$ for $1 \mathrm{~h}$, and the radiolabeled DPPBDT NPs were applied to a size-exclusion column (PD10 column) and eluted with phosphate-buffered saline (PBS) as the mobile phase. The final product was washed out by PBS and passed through a 0.22-ìm Millipore filter into a sterile vial for in vivo PET. Aliquots of the mixture were removed from the solution at predetermined time intervals and filtered through $30 \mathrm{kDa}$ cutoff filters and the radioactivity of filtrates was then measured. The ${ }^{6 \mathrm{Cu}}{ }_{-}$ DPP-BDT NPs were incubated in buffer and run ITLC to analyze ${ }^{6 \mathrm{Cu}}$ dissociation. Samples were assayed in triplicate. Those results showed that ${ }^{6 \mathrm{Cu}}$ was effectively connected to DPP-BDT NPs, and the radiochemical yield was $33.6 \pm 5.9 \%$. The serum stability test of ${ }^{64} \mathrm{u}$-labeled DPPBDT NPs was described in the section of experiments in supplementary file. The nuclide labeled[4Cu]-DPP-BDT NPs were only used in the experiment of in vivo mice PET imaging, and for in vitro and in vivo PAI and other experiments DPP-BDT NPs were used.

\section{Characterization of DPP-BDT NPs}

Nuclear magnetic resonance (NMR) spectra were recorded on a Bruker Ultra Shield Plus $400 \mathrm{MHz}$ spectrometer $(1 \mathrm{H}$, $400 \mathrm{MHz}$; Bruker Electronics, Billerica, MA, USA) using tetramethylsilane as the internal standard. UV-vis-NIR spectra were recorded on a Shimadzu UV-3600 UV-vis-NIR spectrophotometer (Tokyo, Japan). All UV tests were conducted at room temperature. Gel permeation chromatography (GPC) analysis of the neutral polymers was carried out on Shim-pack GPC $-80 \mathrm{X}$ columns with THF as the eluent and polystyrene as the standard at a flow rate of $1.0 \mathrm{~mL} \mathrm{~min}-1$. Transmission electron microscopy (TEM) images were acquired on a HT7700 transmission electron microscope operating at an acceleration voltage of $100 \mathrm{kV}$. Solutions were dropped onto Formvar-graphite-coated copper grids (300 mesh; Electron Microscopy Science, Hatfield, PA, USA) and air-dried for TEM imaging. Dynamic light scattering (DLS) experiments were carried out by using ALV/CSG -3 laser light scattering spectrometers at a scattering angle of $90^{\circ}$. A CONTIN analysis was used to extract the Rh data. The methyl thiazolyl tetrazolium (MTT) assay was conducted using a Power Wave XS/XS2 microplate spectrophotometer (BioTek, Winooski, VT, USA). PA spectra and PA/US coregistered images were acquired with a LAZR instrument (Visual Sonic Vevo 2100 LAZR Imaging System). PET imaging was performed using Inveon micro PET scanner (Siemens Medical Solutions).

\section{Cell Culture}

HepG2 cells and NIH3T3 cells were incubated in Dulbecco's modified Eagle's medium (DMEM) supplemented with 1\% antibiotics (penicillin streptomycin) and 10\% fetal bovine serum (FBS). All cells were incubated in a humidified 
incubator with $5 \% \mathrm{CO}_{2}$, and the temperature maintained at $37^{\circ} \mathrm{C}$, and the medium exchange was operated every $1-2$ days. HepG2 and NIH3T3 Cells were in the logarithmic phase of growth in all experiments.

\section{Cytotoxicity Assay}

NIH3T3 cells were used for the cytotoxicity experiment of DPP-BDT NPs. The cells were incubated in DMEM medium at $37^{\circ} \mathrm{C}$ in $\mathrm{CO}_{2}$ atmosphere for $24 \mathrm{~h}$. Cells were then cocultured with DPP-BDT NPs at different concentrations $(0.01$, $0.1,1,10$, and $100 \mu \mathrm{g} / \mathrm{mL})$. Next, on the basis of vendor's protocol, added $20 \mu \mathrm{L}$ the standard 3-(4,5-dimethylthiazol2-yl-2,5-diphenyltetrazoliumbromide (MTT) into the 96-well plates were incubated for $4 \mathrm{~h}$, removed the mediums containing MTT and replaced with dimethyl sulphoxide (DMSO, 100 $\mu \mathrm{L}$ ). The absorbance of each well was tested by a microplate reader (Infinite M1000, TECAN, USA). A negative control group was tested with a definite volume of saline mixed with culture medium. Cell viability was expressed as the ratio of the absorbance of the cells incubated with DPP-BDT NPs to that of the without DPP-BDT NPs treated cells.

\section{Animals and Tumor Model}

A clear statement of ethical approval for involving animals in the study along with the full name of the ethics committee has been provided in the revised manuscript. This study was performed in strict accordance with the NIH guidelines for the care and use of laboratory animals (NIH Publication No. 85-23 Rev. 1985) and was approved by the Institutional Animal Use and Care Committee of Shan Xi Medical University (Approval No. 2016LL141, Taiyuan, China).

In vivo studies, 5 7weeks, $18 \pm 2 \mathrm{~g}$, athymic female nude mice were purchased from the Beijing Vital River Laboratory Animal Technology Co. Ltd. And housed in sterile cages with enough food and water, and had the constant temperatures $26^{\circ}$ C. HepG2 tumor model was used for in vivo tumor PA and PET imaging. The HepG2 tumor-bearing nude mice were prepared by subcutaneous injection of HepG2 cells $(1 \times 106)$ suspended in $100 \mu \mathrm{L}$ of PBS into the right shoulder of each mouse. The tumor was expected to grow for two weeks, until the tumor volume reached to approximately $100 \mathrm{~mm} 3$. The volume of tumor was calculated by the equation $\mathrm{V}=0.5 \mathrm{LW}^{2}$ $\mathrm{W}$ and $\mathrm{L}$ represent the transverse diameter and longitudinal of the tumor, respectively.

\section{In vitro and in vivo Photoacoustic Imaging} We first studied the PA spectrum of DPP-BDT NPs to acquire the optimal excitation wavelength for subsequent in vitro and in vivo PAI. In brief, we collected the PA signals of DPPBDT NPs activated by a series of excitation wavelength (from $680 \mathrm{~nm}$ to $900 \mathrm{~nm}$ ), respectively. The excitation wavelength corresponding to the maximum PA signal is the optimal wavelength. Besides, we studied different concentrations of DPP-BDT NPs (0 to $200 \mu \mathrm{g} / \mathrm{mL}$ ) and collected their PA signals at the optimal excitation wavelength. As for the in vivo PA imaging, the neoplastic mice were performed anesthetization and injected with the DPP-BDT NPs (200 $\mu \mathrm{L}, 5 \mathrm{mg} / \mathrm{mL}$ ) through systemic injection. Then, we collected the PA signals of the tumor regions at different time points (pre-injection, $2 \mathrm{~h}, 424 \mathrm{~h}$ ). PAI images were operated by a LAZR commercial instrument (2100 High-Resolution Imaging System, VisualSonics). PA/Ultrasound (US) coregistered images were obtained by a MS-250 linear array transducer (20 dB two-way bandwidth, $21 \mathrm{MHz}, 256$ elements).

\section{Small-Animal PET}

The small animal PET imaging of tumor-bearing mice was performed at 2, 4 and $24 \mathrm{~h}$ post-injection on a Siemens Inveon micro PET/CT. Each mouse was injected with ${ }^{6 \mathrm{Cu}}$ radionuclide $(70.0 \pm 5.0 \mathrm{MBq})$ labeled DPP-BDT NPs through the tail vein $(n=3)$. PET scanning was conducted at 2, 4 and $24 \mathrm{~h}$ post-injection (pi). Mouse was anesthetized with isoflurane (5\% for induction and $2 \%$ for maintenance in $100 \%$ O2). Under a laser beam indication of the scanner, mouse was placed in the prone position and near the center of the field of view (FOV) for 5 min static scans. The final whole-body PET images were acquired by the $3 \mathrm{D}$ reconstruction, using Irw4.0 software by two-dimensional ordered-subsets expectation maximization (OSEM) algorithm. Noted that, no attenuation correction was performed. The PET quantitative analysis of the tumor and normal organs was calculated by region of interest drawn, and the intensity was defined as a percentage of the injected radioactive dose per gram of tissue $(\% \mathrm{ID} / \mathrm{g})$.

\section{Histology Analysis}

After 30 days of post-injection, all mice were conducted euthanasia and collected with major organs including lung, spleen, liver, kidney and tumor tissues. And major organs were embedded in optimal cutting temperature (Tissue-Tek ${ }^{\circledR}$ O.C.T.) compound and fixed in $4 \%$ paraformaldehyde, and sliced into $10-\mu \mathrm{m}$-thick, stained with hematoxylin and eosin (H\&E), examined by a digital microscope. The blood hematology and biochemistry analysis were described in the section of experiments in supplementary file. 


\section{Statistical Analysis}

The SPSS 12.0 software was used to analyse the experimental data. PA signal intensities were measured by region of interest (ROI) analysis using OsiriX. Measurement data are expressed as the mean \pm standard deviations (SD). A Student's $t$-test was applied to identify significant differences in cell viability assays and one-way or two-way analysis of variance (ANOVA) was conducted in multiple group comparison. The value of $p<0.05$ was considered statistically significant.

\section{Results and Discussion Properties and Characterization of DPP-BDT NPs}

To prepare DPP-BDT NPs, we first synthesized the organic conjugated polymer DPP-BDT via Stille coupling polymerization according to previous studies, ${ }^{34}$ and its structure was shown in Figure 1A. The polymers possessed a conjugated framework based on the DPP and benzodithiophene BDT units, in which octyl segments were introduced to the DPP and BDT unit, respectively. The DPP unit appeared strongly electron withdrawing and highly absorbing in the visible region, while the BDT possessed planar conjugation, good region regularity and easy modification, which had been served as the most promising donor-electron units for conjugated polymers. With the featured planar and large molecular structure to facilitate conjugated $\pi-\pi$ stacking and charge transfer, BDT and DPP had been acted as electron donor-acceptor polymers. The curve of gel permeation chromatography (GPC) depicted in Figure 1B, the elution time of the DPP-BDT appeared unimodal distribution, and the number-average $\left(M_{n}\right)$ was 29,000 with a comparatively narrow polydispersity index approximately 1.73 , by GPC measurement. The resulting conjugated polymer was further characterized by1H NMR spectra (Figure S1). Those results confirmed that the DPP-BDT was successfully synthesized via a Stille-coupling reaction. The typical amphiphilic molecules DSPE-mPEG5000 were used to encapsulate the DPP-BDT. Upon directly dispersed in aqueous solution, the polymer and amphiphilic molecules rapidly formed into NPs via noncovalent hydrophilic-hydrophobic interaction with hydrophilic parts of amphiphilic molecules as the external shell and hydrophobic DPP-BDT moieties as the internal core. As a result, the DPP-BDT micelles NPs were then constituted by self-assembly process. In order to endow the NPs with the performance of positron emission tomography (PET) imaging, some amount of DSPE-mPEG5000 with macrocyclic chelator NOTA at the terminus can be adopted for radiometa ${ }^{6 \mathrm{Cu}}$ labeling, mainly because NOTA chelator offers efficient chelation with radiometal. ${ }^{6 \mathrm{Cu}}$ As shown in Supporting Figure S2 serum stability studies of64, u-labeled DPP-BDT NPs were subsequently conducted to validate the stability of $[4, \mathrm{u}]$ labeling in vitro and the feasibility for in vivo applications. After incubating with mouse serum at $37^{\circ} \mathrm{C}$ for $40 \mathrm{~h}$, above $95 \%[4 \mathrm{Cu}]$ still remained intact on DPP-BDT NPs at all the tested time points. Since PET imaging detected isotope rather than nanoparticles per se, high radio-stability in serum made4Cu-labeled PPB-BDT NPs preferable for in vivo imaging and truly reflected the distribution of nanoparticle.

Transmission electron microscopy (TEM) imaging manifested the spherical morphology of DPP-BDT NPs with fine homogeneity and monodispersity with the similar average particle diameter of around $31.3 \pm 2.8 \mathrm{~nm}$ (Figure 1D). Moreover, the as-prepared NPs prepared exhibited excellent water-solubility with dark green color, and the hydrodynamic sizes of DPP-BDT NPs were further measured by dynamic light scattering (DLS). The DLS results illustrated that the hydrodynamic radius (Rh) of DPP-BDT NPs was $32 \pm 2.9$ $\mathrm{nm}$ (Figure 1E). The DPP-BDT NPs had remarkable colloidal stability, and no DPP-BDT molecule leaching out of the NPs, which was evidenced by the unaltered size after being incubated in bovine serum PBS buffer at $37^{\circ} \mathrm{C}$ for $48 \mathrm{~h}$ (Figure S3). Such a good colloidal stability of DPP-BDT NPs can be ascribed to the strong $\pi-\pi$ and hydrophobic interactions among planar DPP-BDT molecules in the NPs core and the high-density amphiphilic molecules in the NPs surface.

To further evaluate the DPP-BDT NPs for PAI, we firstly investigated the photophysical capacities of the NPs. As described in Figure 1C, the conjugated polymer NPs possessed a double-peak absorption in the NIR region ranging from 650 to $800 \mathrm{~nm}$. The NPs had the optical absorption of longer wavelength mainly because the DPP electron-deficient element and BDT electron-rich element give rise to the powerful intramolecular charge transfer (ICT) effect. Besides, the short wavelength absorption of the NPs resulted from the $\pi-\pi^{*}$ transition of the semiconducting polymer framework. Importantly, after $60 \mathrm{~min}$ of sustaining laser exposure (Figure S5), PPD-BDT NPs were found to be greatly photostability (only 5\% reduced absorption). In contrast, the FDAapproved indocyanine green (ICG) dye obviously reduced optical absorption (above 80\%) after laser exposure as displayed in Figure S4. Thus, the as-prepared DPP-BDT NPs possessed excellent optical stabilities for biological imaging. 


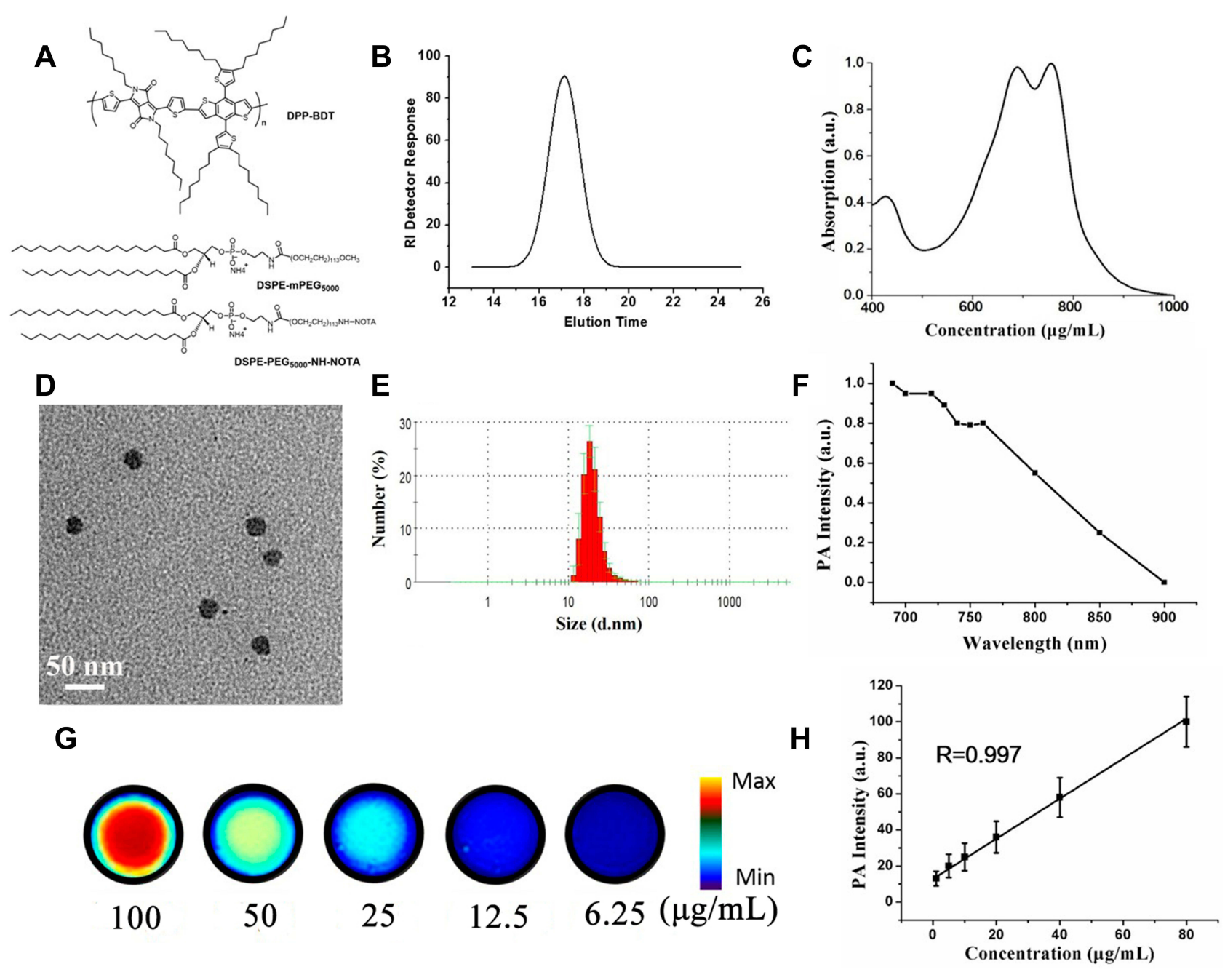

Figure I Characterization of DPP-BDT NPs. (A) Schematic illustrations of the structure of DPP-BDT molecule, DEPE-mPEG5000 and DSPE-PEG5000-NH-NOTA. (B) Chromatogram of RI detector response $(\mu \mathrm{V})$ against elution time $(\mathrm{min}) .(\mathbf{C})$ UV-vis-NIR spectra of DPP-BDT NPs in water. $(\mathbf{D})$ TEM images, scale bar $=200 \mathrm{~nm}$. Inset: aqueous solution of DPP-BDT NPs. (E) Hydrodynamic radius $\left(R_{h}\right)$ of DPP-BDT NPs measured by dynamic light scattering (DLS). (F) PA signal of DPP-BDT NPs at different wavelengths. (G) PAI of DPP-BDT NPs in an agar phantom at concentrations of $6.25,12.5,2.5$, 50 , and $100 \mu g / \mathrm{mL}$. (H) Linear relationship between the PA signal intensity and DPP-BDT NPs at different concentrations.

The PA potencies of DPP-BDT NPs were researched in extracorporal experiment. We first studied the PA spectrum of DPP-BDT NPs to acquire the optimal excitation wavelength for subsequent in vitro and in vivo PAI. From the PA spectrum (Figure 1F), it was clear that the maximum PA signal of PPD-BDT NPs was at $680 \mathrm{~nm}$, which was roughly accorded with its optical absorption spectrum. Hence, $680 \mathrm{~nm}$ was selected as the excitation laser wavelength in our system to obtain optimal PA signals. We further evaluated the PA amplitudes of PPD-BDT NPs at $680 \mathrm{~nm}$ with different NPs concentrations in aqueous solution. Figure $1 \mathrm{G}$ demonstrates the DPP-BDT NPs possessed a bright PA signal contrast and the PA signals of the NPs were linearly increased with the incremental concentration from 1 to $80 \mu \mathrm{g} / \mathrm{mL}$ (Figure $1 \mathrm{H}$ ). Hence, the fine photostability, strong NIR absorption property as well as efficient PA contrast effect of the PPD-BDT NPs made it a promising candidate for in vivo PAI.

\section{The Vitro Cytotoxicity Assay}

The cytotoxicity of DPP-BDT NPs to NIH3T3 cells was assessed by MTT assay (Figure 2A). The cellular activity test indicated that DPP-BDT NPs were not obviously cytotoxic to NIH3T3 cells. DPP-BDT NPs were an excellent biocompatible nanomaterial, and more than $96 \%$ of the NIH3T3 cells remained alive despite being exposed in DPPBDT NPs $\left(0.50 \mathrm{mg} \mathrm{mL}^{-1}\right)$ for $24 \mathrm{~h}$. This result suggested that DPP-BDT NPs were favorable for a variety of biomedical applications. 


\section{In vivo PAI of DPP-BDT NPs}

Inspire by the strong PA contrast potency of DPP-BDT NPs in vitro, the in vivo PAI was further conducted. We studied the tumor accumulation performance of the DPPBDT NPs in HepG2 tumor mice. The HepG2 tumor models were performed through implanting HepG2 cells in the nude mice. After tail vein injection of DPP-BDT NPs, the PA and ultrasound (US) images were recorded for investigating the PAI sensitivity of DPP-BDT NPs. As exhibited in Figure 2B, at pre-injection of the NPs, the very weak PA signal of tumorous region was detected mainly because of the inherent NIR optical absorption of hemoglobin. ${ }^{35}$ The PAI signals at $0 \mathrm{~h}$ can as the control animal without NPs injection. After injection DPP-BDT NPs into mice, the PA signals in the neoplastic tissues gradually increased and reached the peak at 4hpostinjection. Such strong PAI signal observed was attributed to the efficient NPs accumulation resulting from the enhanced permeability and retention (EPR) effect of tumor tissue. At $24 \mathrm{~h}$ post-injection, the PA signals a little decreased while appearing in the whole tumor region, indicating the DPP-BDT NPs had successfully penetrated to inner region of the tumor.

To visually probe the accumulation of DPP-BDT NPs in living mice, PAI was further used to evaluate the biodistribution of DPP-BDT NPs in vivo over time. HepG2 neoplastic mice were performed euthanasia at $24 \mathrm{~h}$ postinjection. The major organs including spleen, liver, heart, intestine, kidney, lung, muscle and tumor tissue were collected and conducted ex vivo PAI (Figure 2C and D). At $24 \mathrm{~h}$ post-injection, we found that the strongest PA signals concentrated in the liver, spleen, and followed by the tumor and kidney, the rest of the organs had shown fewer signals. It was mainly ascribed to the reticuloendothelial system as well as the enhanced penetration and retention effect of tumor.
A

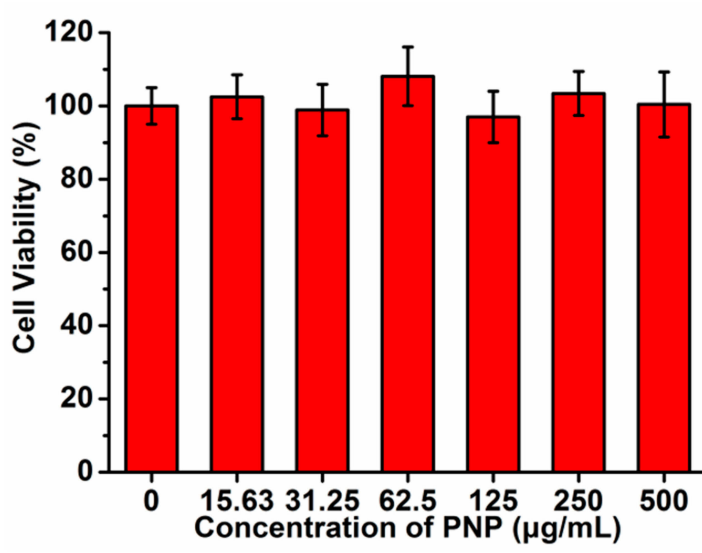

C

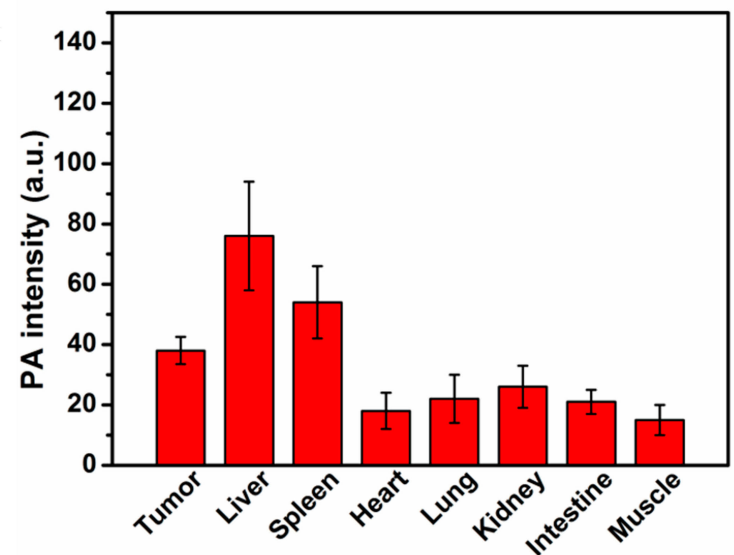

PAI

B

$\mathbf{0 ~ h}$
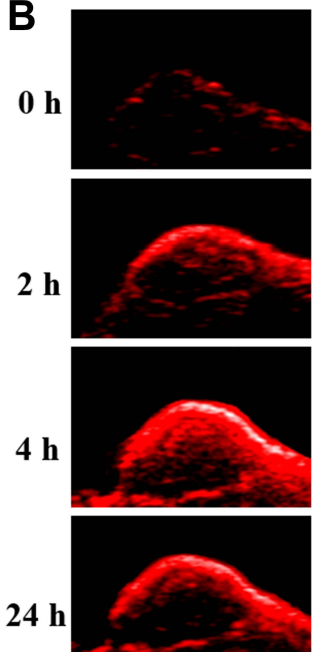

$24 \mathrm{~h}$

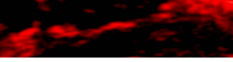

D

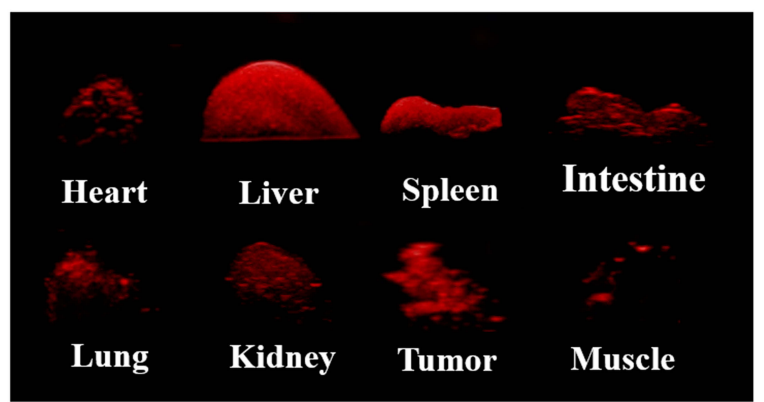

US
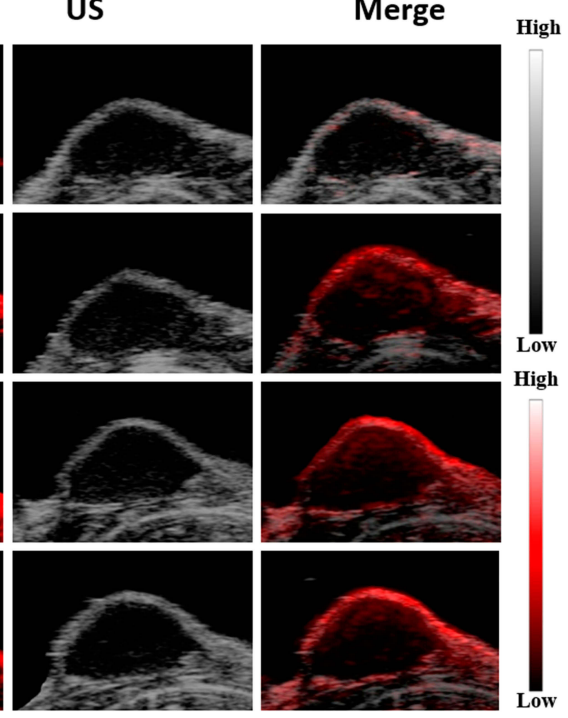

D

Figure 2 (A) The cell viability of the different concentration of DPP-BDT NPs. (B) PA images, ultrasound (US) images, and overlaid PA (red) and US images (grey) of tumor before and after tail vein injection of $200 \mu \mathrm{L}$ of DPP-BDT NPs in living mice (excitation wavelength $=680 \mathrm{~nm}$ for PAl). (C) The histogram between PA intensity and different organs after the mice are sacrificed. (D) The PA intensity of different organs; the size of red areas represent the PA intensity. 


\section{In vivo PET Imaging by ${ }^{6 C u}$-DPP-BDT NPs}

We further investigated the imaging properties of ${ }^{6 \mathrm{Cu}}$-DPPBDT NPs. Herein, the biodistribution and tumor accumulation efficiency of the ${ }^{6 \mathrm{Cu}}$-DPP-BDT NPs in the HepG2 tumor model was demonstrated by dual model imaging of PET and PA. PET imaging can provide whole body scanning of mice to indicate the tumor and quantify the accumulation of ${ }^{6 \mathrm{Cu}}$-DPPBDT NPs in the tumor. For PET imaging, HepG2-tumorbearing mice were intravenously injected with the ${ }^{6 \mathrm{Cu}}$ labeled DPP-BDT NPs (Figure 3A). The tumor and the major organ uptake of the ${ }^{6 \mathrm{Cu}}$-DPP-BDT NPs were calculated by ROI analysis (Figure 3B). A representative PET imaging showed the ROI settings and the distribution of the nanoparticles in Figure S5. The ${ }^{6 \mathrm{Cu}}$ radioactivity in the tumors increased after injection of ${ }^{6 \mathrm{Cu}}$-DPP-BDT NPs and reached to the maximum at the $4 \mathrm{~h}$ post-injection, and then decreased over time. Quantification analysis revealed that the tumor uptake values of $^{6 \mathrm{Cu}}$ were $4.67 \pm 0.59,6.25 \pm 0.47$, and $5.81 \pm 0.67 \% \mathrm{ID} / \mathrm{g}$ at 2,4 , and $24 \mathrm{~h}$, respectively (Figure $3 \mathrm{~B}$ ). In addition to the tumor, abundant ${ }^{6 \mathrm{Cu}}$ radioactivity was detected in the livers $\left(9.19 \pm 2.3 \% \mathrm{ID} \mathrm{g}^{-1}\right)$, spleens $\left(5.79 \pm 3.5 \% \mathrm{ID} \mathrm{g}^{-1}\right)$ and kidneys $\left(5.07 \pm 1.9 \% \mathrm{ID} \mathrm{g}^{-1}\right)$ at $24 \mathrm{~h}$ post-injection, while there was little in the other major organs, indicating the uptake of ${ }^{6 \mathrm{Cu}}$ DPP-BDT NPs in mainly through tumor, kidneys and reticuloendothelial system. Since PET imaging showed different slices, the slice selected in Figure 3 did not include the kidney, so the signal of the kidney cannot be detected. In the supplementary data (Supporting Figure S6), we selected the layer that contained the kidneys, which showed the kidney signals. These results showed that DPP-BDT NPs as the active platform to load ${ }^{6 \mathrm{Cu}}$ can efficiently combine the DPP-BDT's intrinsic photoacoustic properties with radioactive properties together for dual-modality imaging.
PET imaging provides high sensitivity and quantitative tracking of radiotracers, but lacks resolving morphology. PA imaging with deep tissue penetration and high spatial resolution was excellent complementary imaging techniques for PET. Due to the limit penetration of laser, the PA imaging usually monitors probe distribution in a specific organ, likes the tumor, liver, or kidney. We can analyze the DPP-BDT NPs distribution inside of the tumor with PA imaging for more regional details and with PET imaging for more wholebody information. But it is hard to register PA images to PET images for colocalization analysis like scientists rarely merge ultrasound imaging to CT imaging for colocalization. Overall, PET and PAI can serve as an excellent synergistic imaging.

\section{In vivo Cytotoxicity Assay}

The in vivo cytotoxicity of DPP-BDT NPs in mice was further examined through hematoxylin and eosin (H\&E) staining after $14 \mathrm{~d}$ treatment during the study period. Following tail vein injection of DPP-BDT NPs, H\&E staining images of major normal organs did not show any significant damage or inflammation to the heart, liver, spleen, lungs, and kidney in mice from different animal groups (Figure 4). Compared to the control group, the blood chemical and biochemical indicators of all treated mice were almost no changes, suggesting the high biosafety of the DPP-BDT NPs (Supporting Figure S7). The above results suggested that DPP-BDT NPs were an ideal agent with low toxicity for PAI/PET imaging-guided cancer therapy.

\section{Conclusion}

In summary, we successfully designed and prepared an organic DPP-BDT NPs, with good biocompatibility and excellent optical stability, for photoacoustics (PA) and
A

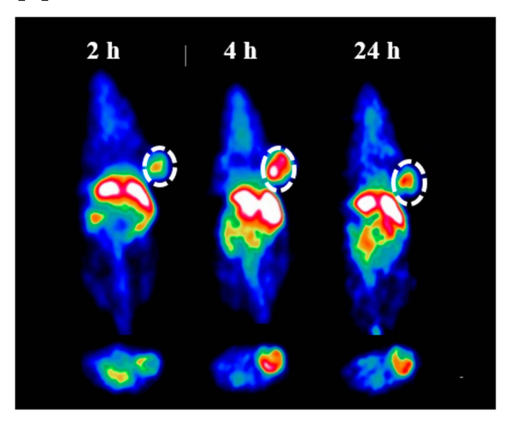

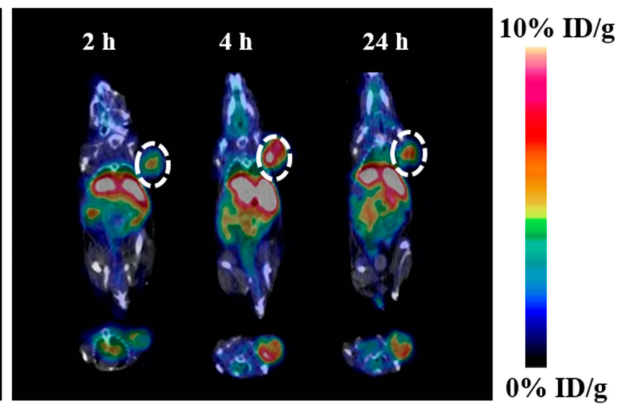

B

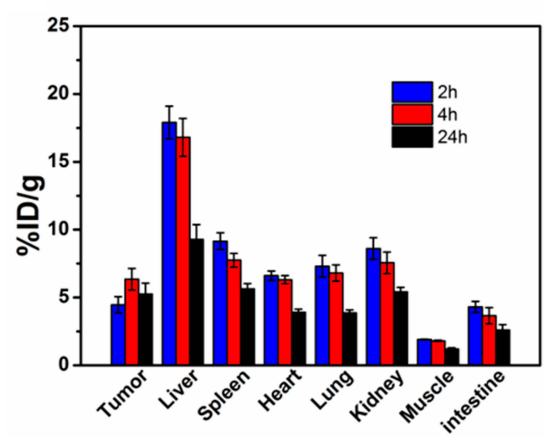

Figure 3 In vivo study of PET of ${ }^{6 C u}$ labeled DPP-BDT NPs. (A) Representative decay-corrected coronal (top) and transaxial (bottom) small-animal PET images (left) and overlaid CT (grey) and PET (color) images (right) of HepG2 tumor (region enveloped by white dotted line) acquired at 2,4 , and $24 \mathrm{~h}$ after tail vein injection of ${ }^{6 C u}$ radiolabeled $(70.0 \pm 5.0 \mathrm{MBq})$ DPP-BDT NPs. (B) The biodistribution of ${ }^{6 C u}$-radiolabeled DPP-BDT NPs in mice $(\mathrm{n}=3)$ at 2,4 , and $24 \mathrm{~h}$ after tail vein injection. The radioactive signal from each organ was calculated using a region of interest drawn over the whole organ region. 


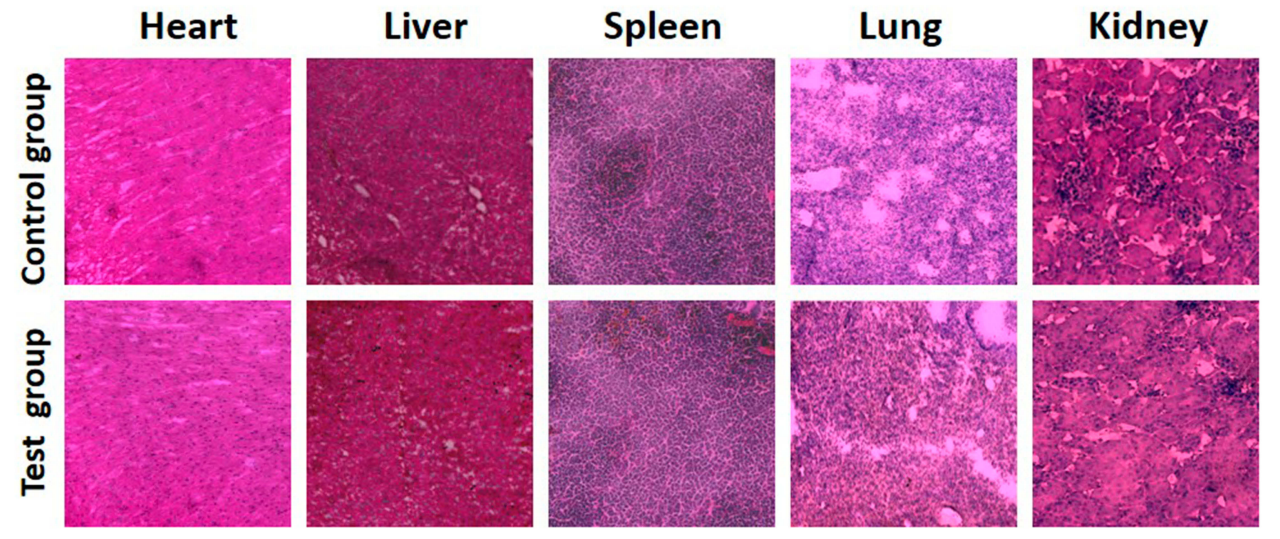

Figure 4 Hematoxylin and eosin (H\&E) staining images of major organs.

positron emission tomography (PET) imaging in living mice. We systematically investigated the biodistribution and accumulation of the DPP-BDT NPs in HepG2 tumor models after intravenous injection by PA and PET imaging. The results revealed that the strongest PA signals in the liver, and next spleen, and followed by tumors and kidney, the rest of the organs had shown fewer signals, which was consistent with the PET analysis. At last, H\&E stained images proved the NPs are an ideal HepG2 tumor nanoagent with low cytotoxicity for PA/PET application.

\section{Funding}

This work has been financially supported by the National Natural Science Foundation of China (Nos. 81571747 and 81771907), Science and technology innovation team project of Shanxi Province (No. 201705D131026), Engineering Technology Research Center of Shanxi Province (No. 201805D121008), Scientific and Technological Achievements Transformation Project of Shanxi Province (No. 201704D131006), Laboratory Construction Project of Shanxi Province, and the Projects for Local Science and Technology Development Guided by the Central Committee (No. YDZX20191400002537). The Fund Program for the Scientific Activities of Selected Returned Overseas Professionals in Shanxi Province, Support from Scientific and Technological Innovation Programs of Higher Education Institutions in Shanxi (STIP), Start up Foundation for Doctors of Shanxi Medical University (No. 03201531), and The Youth Research Fund of Shanxi Medical University (02201618) are also acknowledged.

\section{Disclosure}

The authors report no conflicts of interest in this work.

\section{References}

1. Yang Z, Chen XY. Semiconducting perylene diimide nanostructure: multifunctional phototheranostic nanoplatform. Acc Chem Res. 2019;52:1245-1254. doi:10.1021/acs.accounts.9b00064

2. Liao J, Qi T, Chu B, Peng J, Luo F, Qian Z. Multifunctional nanostructured materials for multimodal cancer imaging and therapy. J Nano Sci Encenanotechnol. 2014;14(1):175-189. doi:10.1166/ jnn.2014.9049

3. Yang Z, Dai YL, Shan LL, et al. Tumour microenvironment-responsive semiconducting polymer-based self-assembling nanotheranostics. Nanoscale Hori. 2019;4:426-433. doi:10.1039/C8NH00307F

4. Cai X, Zhang YS, Xia Y, Wang LV. Photoacoustic microscopy in tissue engineering. Mater Today. 2013;16(3):67-77. doi:10.1016/j. mattod.2013.03.007

5. Lyu Y, Zhen X, Miao Y, Pu K. Reaction-based semiconducting polymer nanoprobes for photoacoustic imaging of protein sulfenic acids. ACS Nano. 2017;11:358-367. doi:10.1021/acsnano.6b05949

6. Miao Q, Pu K. Emerging designs of activatable photoacoustic probes for molecular imaging. Bioconjugate Chem. 2016;27:2808-2823. doi:10.1021/acs.bioconjchem.6b00641

7. Hong $\mathrm{H}$, Yang $\mathrm{K}$, Zhang Y, et al. In vivo targeting and imaging of tumor vasculature with radiolabeled, antibody-conjugated nanographene. $A C S$ Nano. 2012;6(3):2361-2370. doi:10.1021/nn204625e

8. Monica S, Carolyn JA. Molecular imaging of cancer with copper-64 radiopharmaceuticals and positron emission tomography (PET). Acc Chem Res. 2009;42(7):832-841. doi:10.1021/ar800255q

9. Yang Z, Fan WP, Zou JH, et al. Precision cancer theranostic platform by in situ polymerization in perylene diimide-hybridized hollow mesoporous organosilica nanoparticles. $J$ Am Chem Soc. 2019;141:14687-14698. doi:10.1021/jacs.9b06086

10. Yang Z, Song JB, Tang W, et al. Stimuli-responsive nanotheranostics for real-time monitoring drug release by photoacoustic imaging. Theranostics. 2019;9(2):526-536. doi:10.7150/thno.30779

11. Nie LM, Huang P, Li WT, et al. Early-stage-imaging of nanocarrier-enhanced chemotherapy response in living subjects by scalable photoacoustic microscopy. ACS Nano. 2014;8:12141-12150. doi: $10.1021 / \mathrm{nn} 505989 \mathrm{e}$

12. Liu YJ, Nie LM, Chen XY. Photoacoustic molecular imaging: from multiscale biomedical applications towards early-stage theranostics. Trends Biotechnol. 2016;34:420-433. doi:10.1016/j.tibtech.2016.02. 001

13. Hu XM, Tang YF, Hu YX, et al. Gadolinium-chelated conjugated polymer-based nanotheranostics for photoacoustic/magnetic resonance/NIR-II fluorescence imaging-guided cancer photothermal therapy. Theranostics. 2019;9:4168-4181. doi:10.7150/thno.34390 
14. Weber J, Beard PC, Bohndiek SE. Contrast agents for molecular photoacoustic imaging. Nat Methods. 2016;13:639-650. doi: $10.1038 /$ nmeth.3929

15. Nie LM, Chen XY. Structural and functional photoacoustic molecular tomography aided by emerging contrast agents. Chem Soc Rev. 2014;43:7132-7170. doi:10.1039/C4CS00086B

16. Park S, Park G, Kim J, Choi W, Jeong U, Kim C. $\mathrm{Bi}_{2} \mathrm{Se}_{3}$ Nanoplates for contrast-enhanced photoacoustic imaging at $1064 \mathrm{~nm}$. Nanoscale. 2018;10:20548-20558. doi:10.1039/C8NR05672B

17. Song J, Kim J, Hwang S, et al. Smart gold nanoparticles for photoacoustic imaging: an imaging contrast agent responsive to the cancer microenvironment and signal amplification via $\mathrm{pH}$-Induced aggregation. Chem Commun. 2016;52:8287-8290. doi:10.1039/ C6CC03100E

18. Hu XM, Lu F, Chen L, et al. Perylene diimide-grafted polymeric nanoparticles chelated with $\mathrm{Gd}^{3+}$ for photoacoustic/T1 weighted magnetic resonance imaging-guided photothermal therapy. ACS Appl Mater Interfaces. 2017;9:30458-30469. doi:10.1021/ acsami.7b09633

19. Zhang YM, Wang DP, Goel S, et al. Surfactant-stripped frozen pheophytin micelles for multimodal gut imaging. Adv Mater. 2016;28:8524-8530. doi:10.1002/adma.201602373

20. Zheng XP, Shi JX, Bu Y, et al. Silica-coated bismuth sulfide nanorods as multimodal contrast agents for a non-invasive visualization of the gastrointestinal tract. Nanoscale. 2015;7:12581-12591. doi:10.1039/ C5NR03068D

21. Zhang YM, Jeon M, Rich LJ, et al. Non-invasive multimodal functional imaging of the intestine with frozen micellar naphthalocyanines. Nat Nanotech. 2014;9:631-638. doi:10.1038/ nnano. 2014.130

22. Cheng K, Kothapalli SR, Liu H, et al. Construction and validation of nano gold tripods for molecular imaging of living subjects. $J \mathrm{Am}$ Chem Soc. 2014;136(9):3560-3571. doi:10.1021/ja412001e

23. Yi H, Ghosh D, Ham MH, et al. M13 phage-functionalized single-walled carbon nanotubes as nanoprobes for second near-infrared window fluorescence imaging of targeted tumors. Nano Lett. 2012;12(3):1176-1183. doi:10.1021/n12031663

24. Shou K, Qu C, Sun Y, et al. Multifunctional biomedical imaging in physiological and pathological conditions using a NIR-II probe. $A d v$ Funct Mater. 2017;27:1700995. doi:10.1002/adfm.201700995

25. Ge JC, Jia QY, Liu WM, et al. Red-emissive carbon dots for fluorescent, photoacoustic, and thermal theranostics in living mice. Adv Mater. 2015;27(28):4169-4177. doi:10.1002/adma.201500323
26. Liu X, Braun GB, Zhong H, et al. Tumor-targeted multimodal optical imaging with versatile cadmium-free quantum dots. Adv Funct Mater. 2016;26(2):267-276. doi:10.1002/adfm.201503453

27. Kim C, Song KH, Gao F, et al. Sentinel lymph nodes and lymphatic vessels: noninvasive dual-Modality in vivo mapping by using indocyanine green in rats-volumetric spectroscopic photoacoustic imaging and planar fluorescence imaging. Radiology. 2010;255(2):442-450. doi:10.1148/radiol.10090281

28. Yang Z, Dai YL, Yin C, et al. Activatable semiconducting theranostics: simultaneous generation and ratiometric photoacoustic imaging of reactive oxygen species in vivo. Adv Mater. 2018;30:1707509. doi:10.1002/adma.201707509

29. Quli F, Kai C, Zhen Y, et al. Photoacoustic imaging: perylene-diimide-based nanoparticles as highly efficient photoacoustic agents for deep brain tumor imaging in living mice. Adv Mater. 2015;27(5):843-847. doi:10.1002/adma.201402972

30. Lu X, Yuan P, Zhang W, et al. A high water-soluble triblock conjugated polymer for in vivo NIR-II imaging and photothermal therapy of cancer. Polym Chem. 2018;9:3118-3126. doi:10.1039/ C8PY00215K

31. Guo B, Sheng Z, Hu D, et al. Molecular engineering of conjugated polymers for biocompatible organic nanoparticles with highly efficient photoacoustic and photothermal performance in cancer theranostics. ACS Nano. 2017;11(10):10124-10134. doi:10.1021/ acsnano. $7 \mathrm{~b} 04685$

32. Yang Z, Fan W, Tang W, et al. Near-infrared semiconducting polymer brush and $\mathrm{pH} / \mathrm{GSH}-$ responsive polyoxometalate cluster hybrid platform for enhanced tumor-specific phototheranostics. Angew Chem Int Ed. 2018;57:14101-14105. doi:10.1002/anie.201808074

33. Yang $\mathrm{Z}$, Tian $\mathrm{R}$, Wu $\mathrm{J}$, et al. Impact of semiconducting perylene diimide nanoparticle size on lymph node mapping and cancer imaging. ACS Nano. 2017;11(4):4247-4255. doi:10.1021/acsnano. $7 \mathrm{~b} 01261$

34. Dou L, Gao J, Richard E, et al. Systematic investigation of benzodithiophene- and diketopyrrolopyrrole-based low-bandgap polymers designed for single junction and tandem polymer solar cells. J Am Chem Soc. 2012;134(24):10071-10079. doi:10.1021/ ja301460s

35. Hu XM, Zhan C, Tang YF, et al. Intelligent polymer- $\mathrm{MnO}_{2}$ nanoparticles for dual-activatable photoacoustic and magnetic resonance bimodal imaging in living mice. Chem Commun. 2019;55:6006-6009. doi:10.1039/C9CC02148E
International Journal of Nanomedicine

\section{Publish your work in this journal}

The International Journal of Nanomedicine is an international, peerreviewed journal focusing on the application of nanotechnology in diagnostics, therapeutics, and drug delivery systems throughout the biomedical field. This journal is indexed on PubMed Central, MedLine, CAS, SciSearch ${ }^{\mathbb{R}}$, Current Contents ${ }^{\mathbb{R}} /$ Clinical Medicine, $^{2}$

\section{Dovepress}

Journal Citation Reports/Science Edition, EMBase, Scopus and the Elsevier Bibliographic databases. The manuscript management system is completely online and includes a very quick and fair peer-review system, which is all easy to use. Visit http://www.dovepress.com/ testimonials.php to read real quotes from published authors. 